\title{
Impact of Ethnicity on Stroke Care and Outcome in Allen County
}

\author{
Margaret Watkins ${ }^{1}$, Luna Wahab¹, Fen-Lei Chang² \\ ${ }^{1}$ Indiana University School of Medicine, ${ }^{2}$ Parkview Health Neuroscience
}

Background/Objective: Stroke is the fifth leading cause of death in the US and the leading cause of long-term disability for adults. Research has shown that some ethnic groups have worse health outcomes after suffering from an acute stroke. It has been shown that certain ethnic groups used EMS services less and took longer to arrive at the hospital for acute stroke care. Since time is of the essence for stroke treatment, our study may provide insight on factors that may lead to the delay in the acute stroke management.

Project Methods: In this retrospective chart review, we will look at patients who suffered from an ischemic or hemorrhagic stroke. From each patient's electronic medical record, demographic information, time from acute stroke onset to hospital stroke activation, stroke severity, usage of EMS services, duration of hospital stay, co-morbid conditions, and stroke outcome will be recorded. Two-way ANOVA is used for statistical analysis.

Results: Data was not able to be collected at this point. We hypothesize that patients of some ethnic groups have longer delay of ED care initiation from the stroke onset, lesser usage of EMS services, and higher rates of co-morbid conditions. We also hypothesize that these factors are correlated with stroke outcome at the time of hospital discharge. In contrast, the distance between home and nearest hospital with stroke care expertise is not different for different ethnic groups.

Conclusion and Potential Impact: The data collected in this study can further explain why some ethnic groups have worse stroke outcomes than others. If the underlying factors responsible for these differences include factors such as failure to use EMS services, steps can then be taken to provide remedy and to promote better acute stroke outcomes for people of all ethnic groups within our community. 
